\title{
Tight-Binding Molecular Dynamics Simulations on Point Defects Diffusion and Interactions in Crystalline Silicon
}

\section{Tang}

$\therefore \therefore . \quad \therefore \quad \therefore \quad \therefore$ L. Colombo.

T. Diaz de la Rubia

\section{PECEl:}

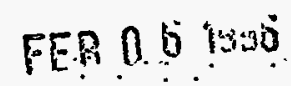

\begin{abstract}
This paper was prepared for submittal to the Materials Research Society 1995 Fall Meeting

Boston, MA

November 27-December 2, 1995
\end{abstract}

OSTI

January 23, 1995

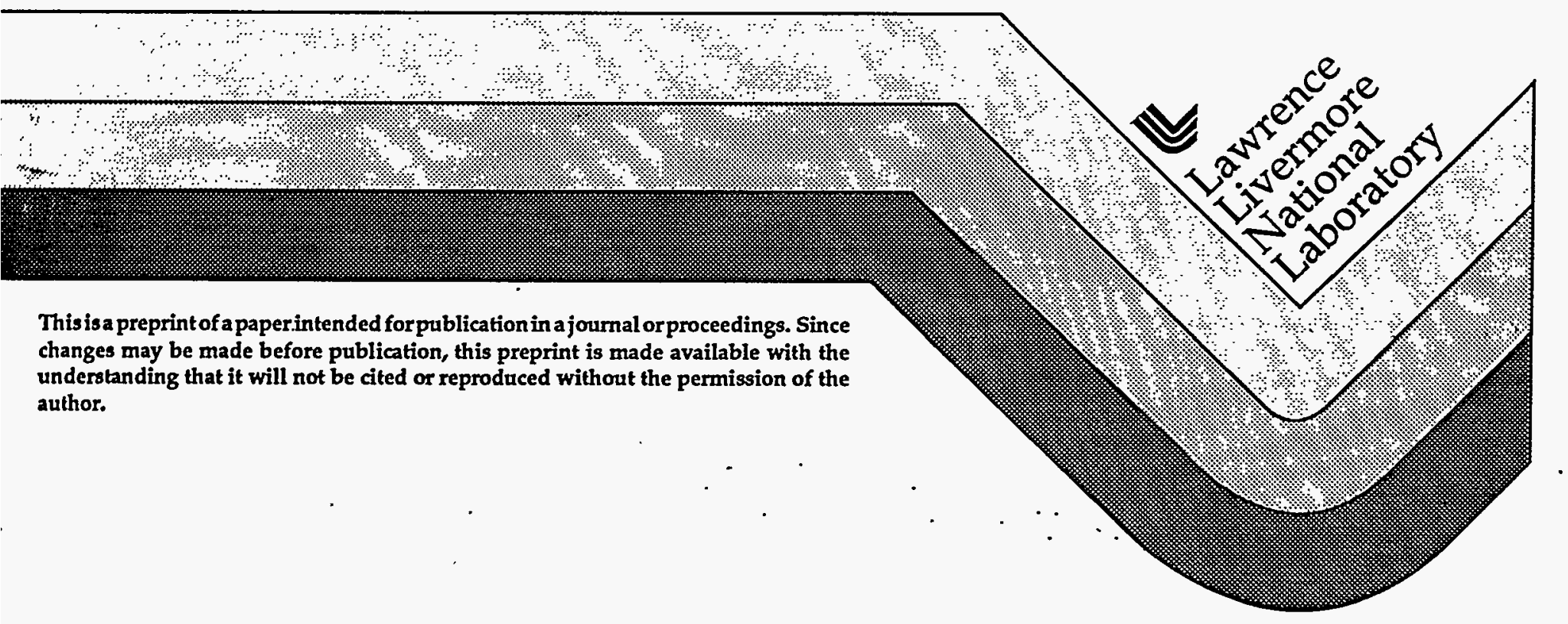




\section{DISCLAIMER}

This document was prepared as an account of work sponsored by an agency of the United States Government. Neither the United States Government nor the University of California nor any of their employees, makes any warranty, express or implied, or assumes any legal liability or responsibility for the accuracy, completeness, orusefulness of any information, apparatus, product, or process disclosed, or represents that its use would not infringe privately owned rights. Reference herein to any specific commercial products, process, or service by trade name, trademark, manufacturer, or otherwise, does not necessarily constitute or imply its endorsement, recommendation, or favoring by the United States Government or the University of California. The views and opinions of authors expressed herein do not necessarily state or reflect those of the United States Government or the University of California, and shall not be used for advertising or product endorsement purposes. 


\title{
TIGHT-BINDING MOLECULAR DYNAMICS SIMULATIONS ON POINT DEFECTS DIFFUSION AND INTERACTIONS IN CRYSTALLINE SILICON
}

\author{
M. TANG ${ }^{\dagger}$, L. COLOMBO ${ }^{\ddagger}, T$. DIAZ DE LA RUBIA ${ }^{\dagger}$ \\ 't Larrence Livermore National Laboratory, P.O.Box 808, L-268, Livermore, CA 9 9550 \\ ; INFM and Dipartimento di Fisica, Università di Milano, via Celoria 16, 20133 Willano \\ (itaiy).
}

\section{ABSTRACT}

Tight-binding molecular dynamics (TBMD) simulations are performed (i) to evaluate the formation and binding energies of point defects and defect clusters, (ii) to compute the diffusivity of self-interstitial and vacancy in crystalline silicon, and (iii) to characterize the diffusion path and mechanism at the atomistic level. In addition, the interaction between individual defects and their clustering is investigated.

\section{INTRODUCTION}

Ion implantation is now a standard technique in the processing of Si-based microelectronics. Further advances in jon beam processing, however, necessitate aststrong improvernent in our basic understanding of defect production, defect diffusion, deject-defect and defect-impurity interactions, amorphization and microstructure evolution. Such an improved fundamental understanding can be obtained by computer simulations, where a validated adranced materials modeling tool is employed. Molecular dynamics (MD) methods are very well suited to provide good models to study irradiation-induced defects. In fact, they offer an atomistic picture of the above phenomena, by describing the complete dynamies of both defects and the host matrix. A crucial issue for MD simulation is the model sdopted for the interatomic interactions. Classical MD is a valuable tool when dealing with collision cascades and subsequent collisions taking place at a long length and time scale. However, fully empirical interatomic potentials have a limited reliability when used to compute formation energies of point-defects, binding energies of clusters of defects or diffusion phenomena. On the other side, first-principle MD simulations (which are more fundamental and accurate) are in practice limited to a small number of atoms (of the order of 100) and to very short simulation times ( ps) berause of their heary computational workload.

In the present rork, we apply tight-binding molecular dynamics (TBMD) to the study of point defects and their clusters in Si. In the TBMD scheme, the forces among atoms are derived from the underlying electronic structure, as in first-principles MD. The electronic structure is, however, computed by means of a semi-empirical tight-binding (TB) model. thus dramatically reducing the computational expense. A detailed description of TBMD can be found elsewhere. $(1,2)$ The TB scheme warrants good modeling of the covalent chemical bonding in crystalline $\mathrm{Si}$. This feature is a key issue when dealing with defects, whose formation and diffusion in a crystalline host matrix result in rather large bosdbreabing and re-bonding.

This paper is organized as follows. We first provide some details of the TBMD model, then present and discuss the results on single point defects and their diffusion, follored by discussions of interstitial and vacancy clusters and final conclusions. 


\section{COMPUTATIONAL METHODOLOGY}

In this rork, we make use of the Kwon et al.(3) TB model for silicon (KBWHS). Here the short-ranged repulsive potential $U_{\text {rep }}$ as well as the scaling functions for the TB hopping integrals are improved compared to the previous Goodwin et al.[4] parameterization (GSP). More specifically, the form of $U_{\text {rep }}$ is embedded-atom like, while in the GSP model only two-body intersction were considered. Furthermore, different scaling functions for the TB hoppings are introduced according to the orbital symmetry in a close agreement to first-principles calculations. The resulting TBMD scheme is more accurate, as demonstrated in Table I that will be discussed in the next section.

Most of the results presented here have been obtained using large cubic periodicallyrepeated simulation supercells containing 216 atoms plus (minus) the number of interstitial (vacancy) defects involved. Typical simulations for annealing and relaxation are performed a few picoseconds. The difiusivities are currently calculated using the 64 atoms cell and with a simulation time up to 100 ps. The formation energy $E^{f}$ is defined as the energy difference between the defected system and the perfect system with same number of atorss. The binding energy for a cluster of size $N$ is defined as $E_{N}^{b}=E_{N-1}^{f}+E_{1}^{f}-E_{N}^{f}$, where $E_{1}^{f}$ is the formation energy of a single defect, and $E_{N-1}^{f}$ and $E_{N}^{f}$ are the formation energies for clusters of size $N-1$ and $N$, respectively.

\begin{tabular}{|c|c|c|c|c|}
\hline Defect & LDA & KBWHS & GSP & SW \\
\hline Vacancy & 3.65 & 3.69 & 3.96 & 2.64 \\
\hline T interstitial & 3.5 & 4.39 & 4.40 & 4.84 \\
\hline H interstitial & 3.3 & 4.93 & 5.90 & 6.58 \\
\hline (110) dumbbell & 3.2 & 3.80 & 5.04 & 3.65 \\
\hline
\end{tabular}

TABLE I - Formation energies ( $\mathrm{eV}$ ) of single point defects in crystalline Si obtained using TBMD of Kwon et al. (KBWHS) and Goodwin et al. (GSP), compared with local density approximation (LDA) results and Stillinger-Weber potential (SW).

\section{RESULTS AND DISCUSSION}

\section{Single Point Defects}

The formation energies of single vacancy and interstitial defects in silicon are calculated by TBMD at low temperature. The interstitiai defects considered are the hexagonal site (H), the tetrahedral site ( $T$ ) and the (110) dumbbell (two atoms share one common lattice site), which are believed to be the most important interstitial defects in crystalline silicon from previous studies. [5-8] The initial structures of each type mentioned abore are set up in a perfect Si crystal, TBMD is then performed to relex the structures until they reach their energy minimum. The formation energies are finally calculated. The vacancy formation energy is computed by taking out one atom from its lattice site and relaxing the crystal to reach its energy minimum. The results of the formation energies are summarized in Table I, and compared to data obtained from first-principle local density approximation (LDA) calculation, GSP and classical (SW) molecular dynamics. The comparisons show that, among the four methods, the formation energy of single vacancy using tise 
TB parametrization by Kron et al. [3] (KBWBS) agrees best xith the IDA calculation. The KBWHS model predicts the same structure as LDA calculation for the energetically most favorable interstitial defect, i.e., the (110) dumbbell, with close formation energies. Note that although the SW model aiso gives close formation energy for (110) dumbbell, the resulting structure is different from LDA results.[5] The bond length of the dumbbell Erom the KBWHS model is $2.41 \mathrm{~A}$, about $10 \%$ smaller than the LDA result.

Although the KBWHS model gives different formation energies for the tetrahedral and bexagonal interstitials when compared to the LDA results, the diffusion path of the (110) dumbbell defect is rather similar to that obtained by IDA calculation.[6] During a TBMD simulation performed at $T=1000 \mathrm{~K}$, the dumbbell found to move into the closest tetrabedral interstitial position along the (110) chain and then move back to a (110) dumbbell at the second nearest neighbor lattice site, as shown in Fig.1. The migration energy for this process can be estimated to be $E^{m} \approx 0.6 \mathrm{eV}$, i.e., of the order of the energy difference between the formation energy of the dumbbell and the tetrahedral interstitial.

(a)

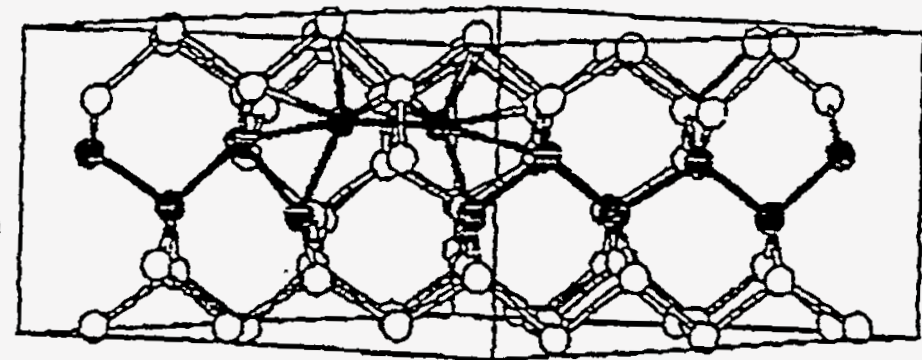

(b)

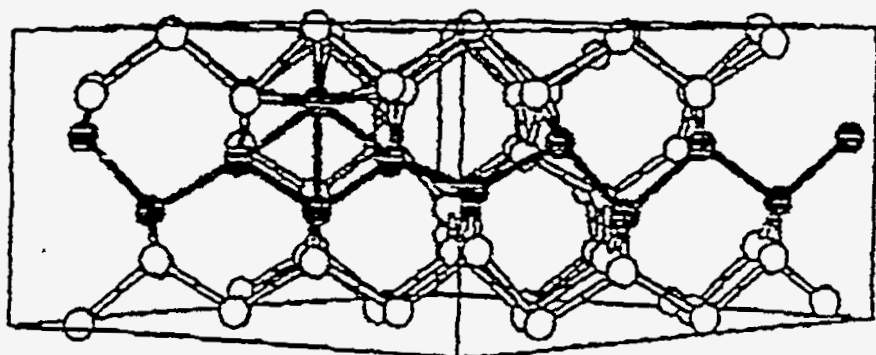

(c)

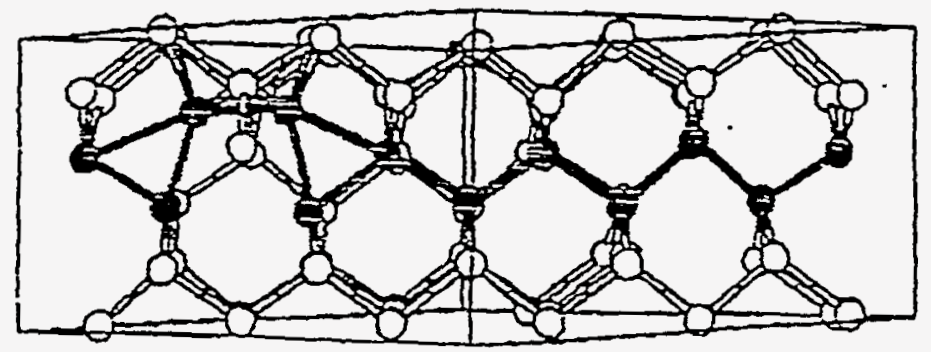

FIG.I - Diffusion path of a (110) dumbbell defect as observed during a TBMD simulation at $1000 \mathrm{~K}$. (a) starting configuration; (b) intermediate configuration. (tbe dumbiell has moved to a tetrabedral position); (c) final configuration (the dumboell structure is recovered at the second nearest neighbor site of (a)). 
Viacancy relaxation shows Jahn-Teller distortions: the four nearest neighbor atoms of the vicancy do not relax symmetrically. One atom moves towards the center of the vacancy by about $40 \%$ of the nearest neighbor distance; the other three atoms move equivalently towards each other, in the near inward direction, to form an equal lateral triangle, zith a displacement of $15 \%$. To roughly estimate the migration energy, the energy difference of a perfect vacancy and a vacancy with $a$ neighbor atom moved to the bond center (which corresponds to the saddle point for diffusion) is calculated, and the migration energy is estimated to be $0.4 \mathrm{eV}$. This is only an approximate value since no relaxation is allowed. A more accurate way to obtain the migration energy is from the Arshenjus plot of the diffusivities, which is in progress.

Experimentally, the self-diffusion coefficients exhibit Arrhenius behavior over a wide temperature range, with an activation energy of $4.8 \mathrm{eV}$. [10] If the diffusion is induced by a mechanism involving either interstitials or vacancies, the activation energy should correspond to the sum of the formation energy and the migration energy of the responsible defect. Based on our calculations, the activation energy for interstitial-mediated and racancy-mediated diffusion is $3.8+0.6=4.4 \mathrm{eV}$ and $3 . \hat{\imath}+0.4=4.1 \mathrm{eV}$ respectively.

Finally, we have computed the diffusivity of the vacancy and dumbbell dejects at finite-temperature of $\sim 1500 \mathrm{~K}$, obtaining $\sim 710^{-7} \mathrm{~cm}^{2} \mathrm{~s}^{-1}$ and $\sim 510^{-8} \mathrm{~cm}^{2} \mathrm{~s}^{-1}$ respectively. These values are smaller thas the SW data reported in Ref.[5].

\section{Interstitial Clusters}

Individual isterstitial defects can interact with esch other during the damage annealing process after ion implaniation to form defect clusters. Under large dose irradiation conditions, they can further develop extended microstructures such as $\{311\}$ defects and dislocation loops. In order to understand the role of defects in transient enhanced diffusion, it is of great importance to study their formation and binding energies.

We have studied a di-intersititial cluster formed through the interaction of two (110) dumbbells. The simulations show that two (110) dumbbells, either parallel or perpendicular to each other, at nearest neighbor sites attract and form a stable configuration given by one (110) dumbbell plus a neighboring tetrahedral interstitial. This di-interstitial has a formation energy of $E^{S}=5.44 \mathrm{eV}$ and a binding energy of $E_{2}^{b}=2.17 \mathrm{eV}$. The diinterstitial cluster found here is different from that obtained using the SW potential, where the di-interstitial is formed by two tetrahedral interstitials at nearest neighbor sites with a binding energy of $1.6 \mathrm{eV}$.(5) The latter di-interstitial structure is found to be unstable in our TBMD and found to change quickly into the stable configuration of one (1:0) dumbbell with a neighboring tetrahedral interstitial at $300 \mathrm{~K}$.

Finally, tri- and tetse-interstitial clusters have also been studied. The tri-interstitial cluster is found to be formed by two perpendicular (110) dumbbells (slightly distorted) sharing a common lattice site ryith a binding energy of $1.68 \mathrm{eV}$. And the tetra-interstitial cluster is found to be iormed by two di-interstitials at nearest neighbor sites with a binding energy of $0.83 \mathrm{eV}$. The study of larger size interstitial clusters is in progress.

\section{Vacancy Clusters}

The vacancy clusters are formed by taking atoms away from adjacent sites in the Si crystal. The system is then allowied to relar and reach its energy miminum to obtain the binding energy. Fig.2 shows the binding energies compared to those from the SW calculation, [5] as n'ell as an experimental data point for the di-vacancy.[9] The di-vacancy: binding energy obtained from our TBMD calculation is in close agreement with the experimental data. An important feature shown in both curves is that the binding energy 
reaches a maximum at a certain cluster size, 4 for KBWHS and 5 for SW. This implies that the vacancy clusters with this particular size are most stable during the defect annealing process. Finally, we would like to point out that, based on our obserrations, the strain field of the vacancy and its clusters are more extended compared to those of the interstitials.

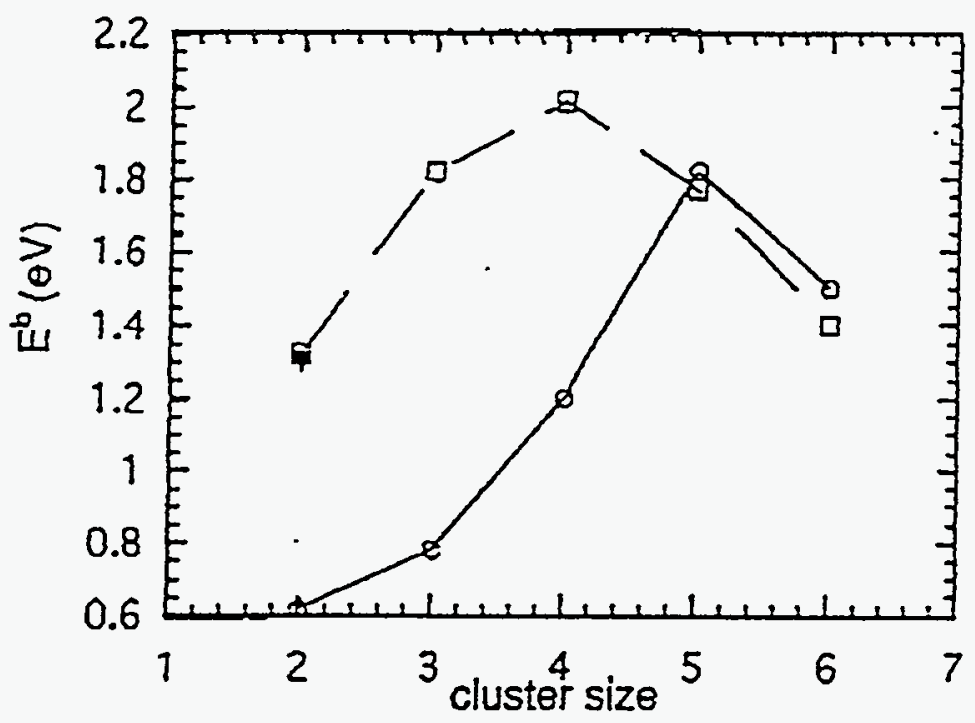

FIG.2 - TBMD binding energy $E^{b}(\mathrm{eV})$ for a vacancy cluster as function of the cluster size (open squares). Experimental (full triangle) and $S W$ (open circles) data are shown for comparison.

\section{CONCLUSIONS}

Based on our results presented above and the comparisons with LDA calculations and available experimental data, we find that the tight-binding KBWHS model provides a valid tool to study teh ground state of neutral point defects in crjstalline silicon. Using this model, fie are able to not only characterize the static structures of point defects and their clusters, but also to describe ther dynamic diffusion in crystalline silicon at atomistic level. A striking observation from the interstitial cluster studies is that the binding energy goes down as the cluster size goes up. This naturally raises the question regarding the formation of large interstitial clusters, such as the extended defect $\{311\}$ observed in experiments. This points to a direction of our future study, i.e., to understand how the $\{311\}$ and other extended defects are nucleated in crystalline silicon.

\section{ACKNOWLEDGEMENTS}

We acknowledge stimulating discussions with G. H. Gilmer and J. Zhu. 'One of us (MIT) acknowiledges discussions with C. Z. Wang. The work is performed under the auspices of the U.S. Department of Energy by Lawrence Livermore National Laboratory under Contract No. WW-7405-Eng-48. One of us (LC) acknowledges CILEA (Milano, Italy) for computational support under project "Fisica computazionale dei Materiali". 
REFERENCES

1. L. Colombo, Proceedings of Physics Computing '94, R. Gruber and M. Tomassini Eds. (22-26 August 1994).

2. C. Z. Wang and K. M. Ho, Comp. Mat. Sci. 2, pp.93 (1994).

3. I. Kwon, R. Biswas, C. Z. Wang, K. M. Ho, and C. M. Soukoulis, Phys. Rer. B 49, 7242 (1994).

4. L. Goodwin, A. J. Skinner, and D. G. Pettifor, Europhys. Leit. 9, 701 (1989).

5. G. H. Gilmer, T. Diaz de la Rubia, D. M. Stock and M. Jaraiz, Nucl. Instr. and Meth. B 102, 247 (1995).

6. J. Zhu, L. H. Yang, C. Mailhiat, T. Diaz de la Rubia, and G. H. Gilmer, COSIRES'94 proceedings, Nucl. Instr. and Meth. B 102, 29 (1995), and private communication.

T. R. Car, P. Blochl, and E. Smargassi, Mater. Sci. Forum 83-87, 433 (1992).

8. P. J. Ulgar, T. Halicioglu, and W. A. Tiller, Phys. Rev. B 50, 7341 (1994).

9. G. D. Watkins and J. W. Corbett, Phys. Rev. 138, A543 (1965).

10. G.U. Gosele and T. Y. Tan, Defect and Diffusion Forum 83, 189 (1992). 

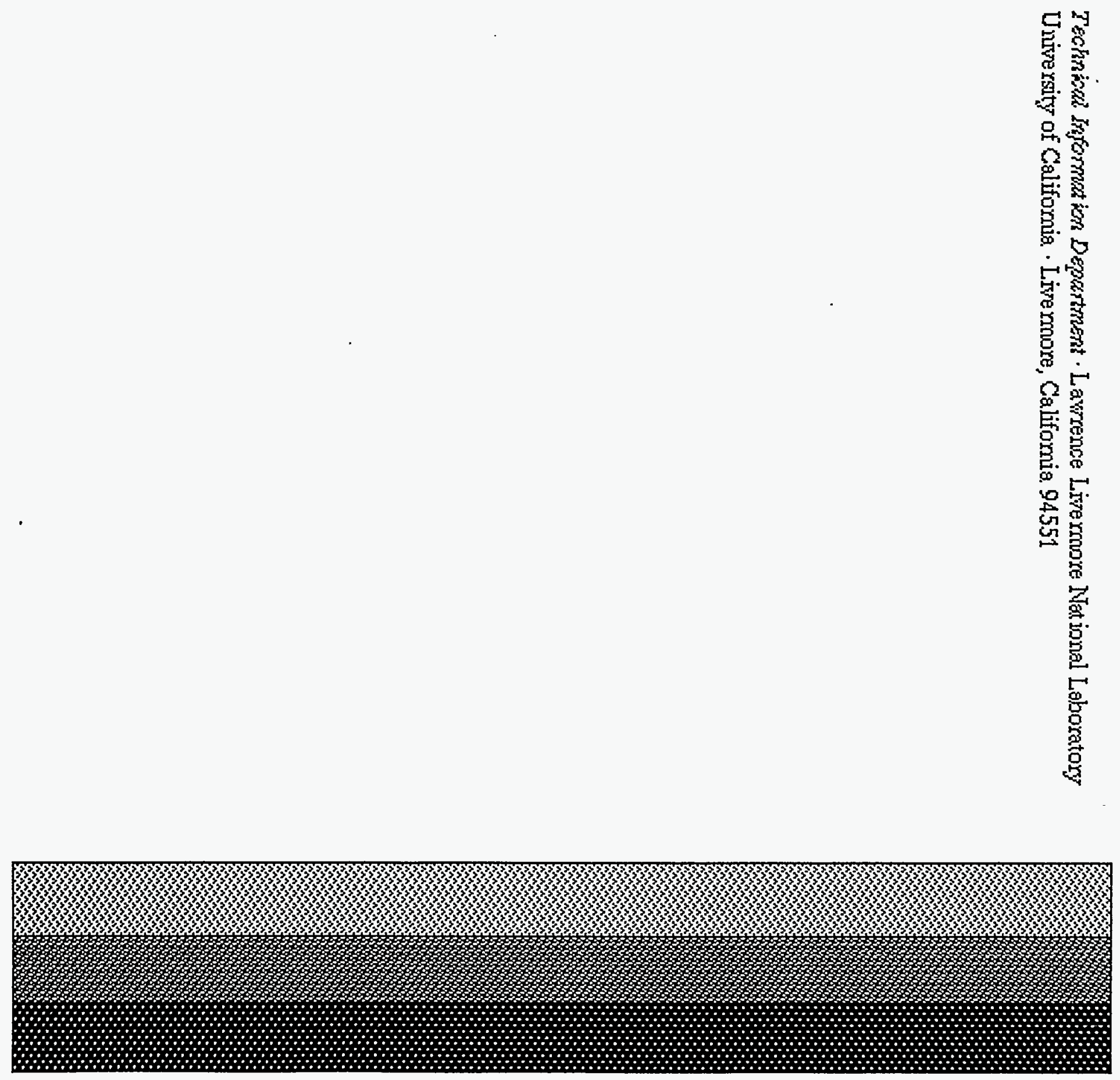